\title{
The panorama between COVID-19 pandemic and Artificial Intelligence (AI): Can it be the catalyst for Society 5.0?
} \author{
Latiff $^{5}$ \\ ${ }^{1} \mathrm{PhD}$ Candidate \\ Putra Business School (AACSB-Accredited) \\ ${ }^{2}$ Masters Candidate \\ Putra Business School (AACSB-Accredited) \\ ${ }^{3} \mathrm{PhD}$ Candidate \\ Putra Business School (AACSB-Accredited) \\ ${ }^{4}$ Professor (Director-Thesis Based Programme) \\ Putra Business School (AACSB-Accredited) \\ ${ }^{5}$ Senior Lecturer \\ Putra Business School (AACSB-Accredited)
}

Ariful Islam¹, Mahmuda Islam², Md. Uzir Hossain Uzir ${ }^{3}$, Sazali Abd Wahab ${ }^{4}$, Ahmad Shaharudin Abdul

\begin{abstract}
Purpose: During the COVID-19 pandemic, a new age digital era has emerged; Artificial Intelligence (AI) could be instrumental in keeping this new virus within reasonable limits. AI also has the potential to transform communities into super smart Society 5.0. So, the main target of the study is to analyze AIfocused Society 5.0 configuration through the lens of the present pandemic.
\end{abstract}

Design/Methodology/Approach: A systematic analysis of literature has been executed to discuss the AIfocused Society 5.0 configuration triggered by pandemic, in which over 300 publications are read and reviewed by the authors. The reliability and validity of literature analysis have also been established.

Findings: The analysis confirms that the COVID-19 emergency can be one of the most important drivers, influencing transformation to Society 5.0 through the implementation of AI. This pandemic sets the perfect platform for global adoption of AI-based Society 5.0 to obtain more ethical human and technology collaboration.

Research limitation: Prior empirical attempts are subsequently required to inquiry about the proposed conceptualization from different perspectives.

Practical implications: The findings will act as a realistic roadmap for applying AI technologies to Society 5.0 outline by the incorporation of open source technologies, supportive regulations, and modern business models. Potential approaches emerging from this research are useful to community managers or large corporations partnering with local agencies to increase the opportunities for the long-term success of smart city projects.

Social implications: The outcomes of the study will help to solve social and ecological issues aligned with SDGs. It will also create an innovative platform to utilize the working abilities of older segments of society.

Originality/Value: This article is the first attempt to discuss AI-focused super intelligent society configuration to harness the hidden potential of the COVID-19 pandemic.

Keywords-Artificial Intelligence, Big-data, COVID-19, Internet of Things, SDGs. 


\section{Introduction}

Our planet has been altered forever by coronavirus disease (COVID-19) from Wuhan, China. The pandemic has spread from Asian to European regions and then to the rest of the globe in a distinctive sequence (Sokolov, 2020). The leaders of several countries agreed to save lives before saving the economy in response to the emergency, declaring abrupt or staggering lockdowns in their nations. Policies such as "communal distancing" and "stay-at-home" were enforced overnight, seriously affecting numerous commercial activities around sectors (Donthu and Gustafsson, 2020, Gupta et al., 2020). The COVID-19 pandemic has, according to Bogler et al. (2020), led to global socio-technical concern and many alternate futures. It is undermining current practices internationally, exponentially, and pervasively (Huynh, 2020) and is rapidly evolving as a catalytic and meta-transition event that threatens and reshapes the validity and usefulness of existing communal systems (Jetten, 2020; Hu, 2020). A strong change is caused by the latest pandemic in which the ways of consumption, trade finance, doing industry, and controlling Governments are transforming through the digital transition of economies.

Digital innovations are being exploited at this stage to support the community's reaction to COVID-19 (Ting et al., 2020). This scenario involves emerging technology based on Artificial Intelligence (AI) to allow suitable responses and analyses, the rapid provision of information on disease dynamics, and an understanding of disease development rules to provide timely guidelines for preventive and control decisions and interventions (Naudé, 2020). In the battle against COVID-19, new intelligent defense strategies have been implemented by many Governments (Herath and Herath, 2020). The global Artificial Intelligence industry is projected to rise from $\$ 28.42$ billion in 2019 to $\$ 40.74$ billion in 2020 at a compound annual growth rate (CAGR) of 43.39 percent (The Business Research Group, 2020), leading to the convergence and streamlining of digital networks at different levels. Microsoft, for example, has invested about $\$ 1$ billion in a San Francisco firm called OpenAI. The latest Gartner Inc (2020) study also shows that since the beginning of COVID-19, 66 percent of companies have raised or not changed AI investments. In addition, 75 percent of this report's respondents will continue or launch new AI schemes as they step into the post-pandemic reset of their organization's renewal process. Again, IDC (2020) reveals an unprecedented Artificial Intelligence (AI) budget of $\$ 10$ billion for 2020, taking into account European areas, and a healthy rise of percent CAGR in 2023. Media, Federal/Central Government, and Technical Services are the areas that will see the most significant growth in AI spending over the 2020-2024 outlook. We would therefore argue that the enormous humanitarian, social, and economic needs posed by COVID-19 are accelerating, at scale and pace, the growth and adoption of new AI-centric emerging technologies. There was tremendous interest in the capabilities of AI even before COVID-19 reached, and the occurrence of the pandemic only helped to strengthen the technology's importance. In line with the above scenario, fiscal, social, and technical shifts would become inevitable for survival if the COVID-19 crisis continues to affect personal and professional activities in the next few years (Verma and Gustafsson, 2020).

In the battle against the recent pandemic, national and local authorities around the world have deployed intelligent smart society concepts to track the spread of the virus and endorse the adoption of medical interventions (Jaiswal et al., 2020). These new applications are starting to shape the future of smart and intelligent human-centered societies, as well as highlighting the importance of AI-based technology. Although the COVID-19 disturbance may momentarily curtail development plans, the long-term outlook is optimistic in relation to the global community's smart transformation. The COVID-19 pandemic has prompted mankind to reconsider the implementation of Japan's Society 5.0 configuration at this stage (DeWit et al., 2020; Muhthar et al., 2020). The outline will help uniform ethical guidelines to minimize the spread of deadly pandemics, facilitating smooth coordination. Japan's adaptive strategies focused on Society 5.0 demonstrate the rapid resilient mechanism and accomplishment of Sustainable Development Goals (SDGs) can be included in the pandemic response (DeWit et al., 2020). Japan is one example of how COVID-19 countermeasures are being used under a smart configuration to bridge national and international imperatives.

Because of its recent effect on human culture, social science analysts and other scholars have now started to investigate the implications of the COVID-19 emergency. The outbreak offers a remarkable opportunity to 
explore the real-time effect of AI in super-smart societies on the different directions of change. Recent studies by Budd et al., 2020, Kanda and Kivimaa, 2020, Islam and Wahab, 2020, Nazir et al., 2020, and Ting et al., 2020, however, addressed the connection between the emergency of COVID-19 and the growth of advanced digital technologies, while only a few research activities studied the structure of AI and Society 5.0 under a globalized smart crisis resistant community context (Fukuda, 2020). In view of the interplay between future science expeditions inside COVID-19, AI, and Society 5.0, only a limited number of scholars have been able to systemize an appropriate path leading to shaping the future of human-focused super-smart societies (Tashiro and Shaw, 2020). At this juncture, we argue that with a future intelligent human-centered view, the COVID-19 emergency could hold the seeds of global adoption of Japan's Society 5.0 roadmap. The recent pandemic can be classified as a black swan event that created a ripple effect on some regions of culture or human activity. Research into the parallel implementation of the effect of COVID-19 enhanced AI on the outline of Society 5.0 contributing to UN's SDGs is also in its infancy (Donthu and Gustafsson, 2020). Therefore, our research goal is to further investigate how the COVID-19 pandemic will promote a stable global system focused on Artificial Intelligence (AI) for Society 5.0 architecture to address these research limitations. It is expected that the range of understandings discovered will further facilitate the conceptualization of this configuration to guide politicians, service agencies, ICT experts, smart city developers, and academics in the sustainable achievements leading to the intersection of digitalization and sustainability.

Holy book Al-Quran in 2:216 (Murad, 2014) says-

\section{"You may dislike something although it is good for you, or like something, although it is bad for you"}

The verse reflects the major underlying foundation of this study. Everything happens for a cause and with precise wisdom. Dark days basically offers the brightest gifts. Compatible with the essence of the verse, we anticipate that COVID-19's sufferings or turbulences will help the probabilities associated with advanced digital technologies that contribute to an intelligent society ecosystem that guarantees quality of life for everyone and facilitates the process of ethical innovation. However another motivation of this paper is to add to the continuing pool of transdisciplinary solution studies that contribute to a modern socio-technical paradigm. According to Jahn et al. (2012) and Max-Neef (2005), transdisciplinarity can be interpreted as a process that incorporates diverse analytical and deeper research viewpoints to contribute to communal progress.

This study is the result of a comprehensive literature review that analyzed the peer-reviewed literature published in emerging digital technology, smart city, Society 5.0 concept, COVID-19, and Sustainable Development nexus in highest impact factor articles. More than 300 papers were read and examined by the authors of this paper. In addition, papers were chosen using Web of Science, Scopus, MDPI, Springer, Emerald, and other respectable databases. This mix of types of databases helped us to compile a detailed list of articles that were important. All papers were grouped into the following five categories: (i) keywords; (ii) research unit; (iii) region of data collection process (i.e., settings); (iv) approach (conceptual vs. empirical); and (v) theoretical implementation. Some AI and digital transformation related reports have been used as secondary sources. Reliability was targeted in this literature review by discussing the methodological steps of a literature review provided by Hart (2001). Three researchers conducted the literature review and each of the measures was addressed before and after to improve inter-rater reliability during the study of literature (Seuring and Müller, 2008). Validity was pursued by sampling papers based on existing criteria (Hart, 2001), and constructs were contrasted inside and outside the field of study from previous published literature reviews (Collin et al., 1996; Fagerberg et al., 2012). Also besides, continuing academic work on this analysis was also discussed at conferences and workshops, encouraging other scholars to comment on the work-inprogress process.

\section{Artificial Intelligence (AI)}

Artificial Intelligence (AI) is a general branch of computer science interested in the development of smart machines capable of executing tasks that usually require human intelligence (Dobrev, 2012). In this concept, the main concern being that the amount of AI intelligence is comparable to a human being's level of intelligence. AI is essentially an interdisciplinary science with various approaches, but in almost every field 
of the software industry, developments in machine learning and deep learning are causing a paradigm change. In a limited context, the analysis of techniques by enhanced programming configuration to use machines more efficiently. In the other hand, Artificial Intelligence is considered the analysis from which expert systems are extracted (Simmons and Chappell, 1988). In research on whether computers could make decisions, AI started to be quoted in the 1940s (Buchanan, 2005). More recently, AI studies and implementations have covered many public sector roles in the 2010s (Wirtz et al., 2019), including but not limited to public health (Sun and Medaglia, 2019), transport (Abduljabbar et al., 2019), education (McArthur et al., 2005), defence (Ku and Leroy, 2014), communications (Letaief et al., 2019), and also the armed forces (Masuhr, 2019). In reality, deep learning technologies in the intellect of machines and robotics have created profound disruptive and empowering effects on industry, politics, and society (Goralski and Tan, 2020). They also affect the broader global sustainability trends (Islam et al., 2020). We conclude that AI will respond favourably to humanity's environmental and social issues as it provides sophisticated tools to achieve the objectives of sustainable development.

\section{COVID-19 pandemic and rise of AI}

AI-based digital technologies soon became the cornerstone of personal and professional life during the COVID-19 pandemic (Panigutti et al., 2020). Digital devices that are connected allow for both remote work and schooling. Also besides, intelligent chatbots have immediate information that saves lives, partly relieving overloaded health systems (Miner et al., 2020). Again, intelligent information systems for geolocation are allowing health workers and researchers to track and chart the distribution of the virus. In order to incorporate emerging technology, such as data policy, digital commerce, IoT, AI, drones, autonomous vehicles, blockchain telemedicine, and environmental developments, corporations and policymakers are developing and piloting quick, sensitive frameworks (Barnes, 2020; Panigutti et al., 2020). In fact, instead of flesh-and-blood spooks, many Governments are now depending on sensors and strong algorithms (Whitelaw et al., 2020; Woo, 2020).

At the time of this crisis, the primary challenge now is to incorporate and streamline AI-based digital technology at different stages of the public health or communal response, particularly in the sense of disease predictions and decision-making. Trials are underway to build and build smart devices that rely heavily on AI to control epidemics (Albzeirat et al., 2020). To monitor the dissemination of diseases in terms of when and how (Ye, 2020), the incorporation of data through the use of AI applications regarding the incubation time, the path of transmission, the ports of entry, travel and cultural activities could be crucial (Ye, 2020). In reality, Artificial Intelligence (AI) is an upcoming and valuable method for detecting early coronavirus infections and also helps to track the status of the patients infected. By designing effective algorithms, medication quality and decision-making can be greatly improved (Vaishya et al., 2020). It may also track the COVID-19 crisis at various stages, such as medical, molecular, and epidemiological applications (Greenspan et al., 2020). AI aims to develop proper care regimens, preventive methods, medication and vaccination production in the base.

\section{The concept of Society $\mathbf{5 . 0}$}

In the fifth step introduced by Japan (Fukuda 2020), Society 5.0 is the concept of a super smart humancentered society. It is the solution (Mavrodieva and Shaw, 2020) to the imminent technical advancement and expanded use of AI-focused Big-data Analytics, Internet of Things (IoT), and Robotics. The definition of Society 5.0 is similar to Industry 4.0 , but takes a step ahead, representing a smart and intelligent configuration, a data-driven economy and society, with an emphasis on human desires and skills. The proposal envisages a fusion of the actual (physical) world with cyberspace in order to gather more reliable and customised data effectively for enhanced problem solving and value generation (Fukuyama, 2018). As per this overview, the massive volume of data that until now required a huge amount of time and resources is now intended to be processed much faster by AI and converted into easy-to-understand knowledge that can be used by humans in business activities and social services (Polat and Erkollar, 2020).In Society 5.0, without human involvement, the process will be driven; humans will only obtain greater opportunities to access information obtained from AI, the final performance of the transfer process (Deguchi et al., 2020). It is predicted that this transition will transform the way culture operates in nearly all aspects of human life and will have a positive effect on the economic growth of many regions, as well as help solve a variety of social 
or ecological problems (Fukuda, 2020; Fukuyama, 2018; Rochman et al., 2020). At this point, the authors of this study also support that Society 5.0 is the mark attached to a vision of a whole strategy of authority, industry and education' to incorporate modern technical structures for the good of humanity across diverse fields.

\section{Figure-1: Japan's Society 5.0}

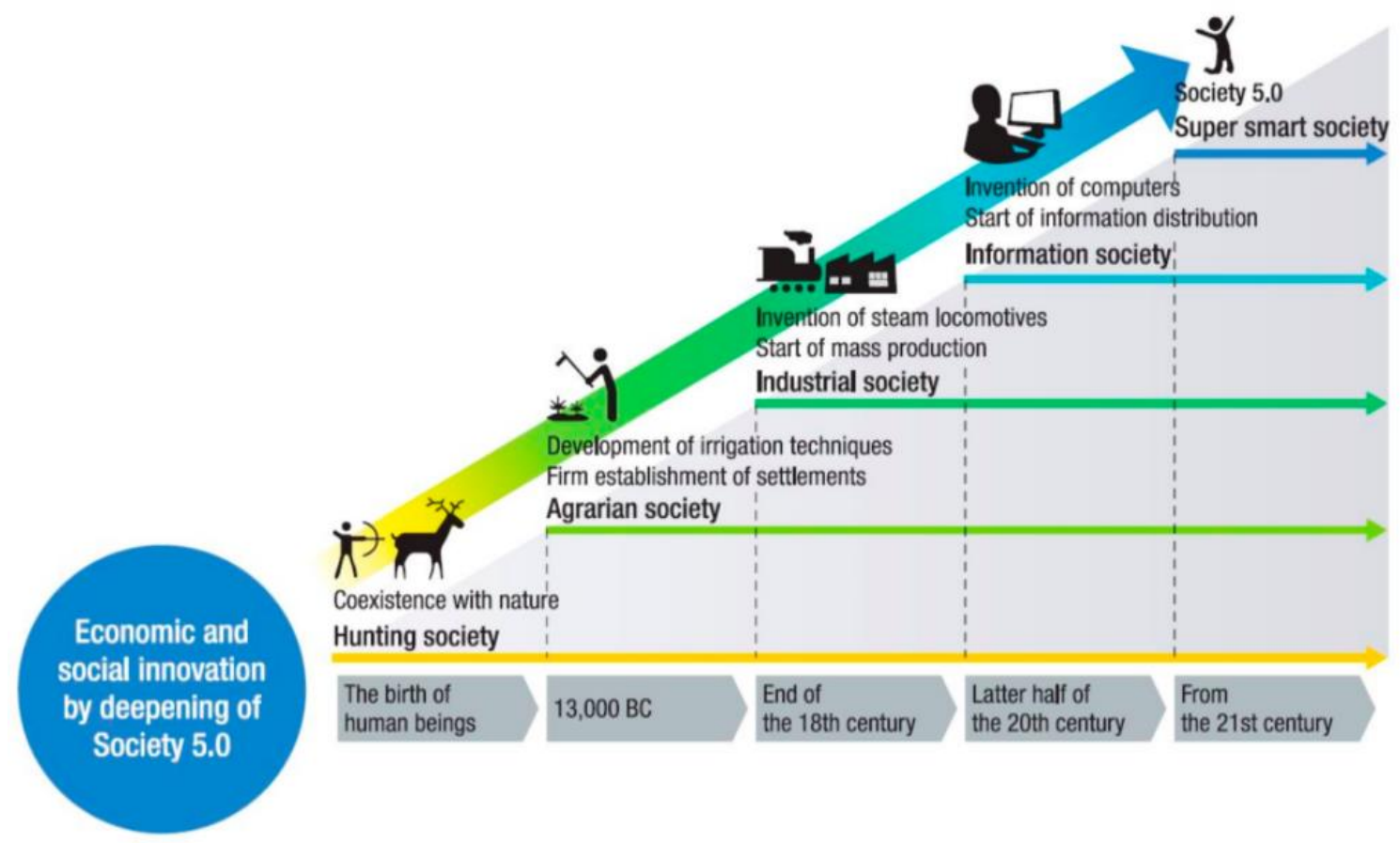

Source: Fukuyama (2018).

When it was included in the 2016 5th STBL (Science and Technology Basic Plan), proposed by the Council of Science, Technology, and Innovation (Fukuda, 2020; Holroyd, 2020), the Society 5.0 outline was made Japan's official policy for the first time. The strategy was negotiated and organized with members of the private sector and the Japan Business Federation-Keidanren and its member firms, most of which are major large corporations, have widely endorsed it. This close cooperation culminated in the establishment of Industry-Government Committees working on designing approaches to the realisation of Society 5.0 in their sectors of focus. At this point, both stakeholders have promised that the current vision for the future can greatly help the accomplishment of the Sustainable Development Goals (SDGs) of the United Nations and ensure that a sustainable, equitable and human-centered society is established (Deguchi et al., 2020; Di Vaio et al., 2020). More recently, Growth Strategy related action plans from 21 June 2019 presented some specifics of the Japanese Government's vision of adopting Society 5.0 in the light of compliances with the SDGs and the Paris Agreement of 2015 (Mavrodieva and Shaw, 2020). Again, Japan's actions are transforming into a holistic communal paradigm in light of the COVID-19 pandemic effect (DeWit et al., 2020). For one thing, they are part of the industrial policy regime of an interconnected, resilience-oriented "Society 5.0" that predated COVID-19 (Mavrodieva and Shaw, 2020). The policy weapons of Society 5.0 include such vital cyber-physical connections as next-generation communications from "post 5G" and remote sensing for catastrophe risk mitigation. Japan also plans to spread this idea globally, however, and collaborate on its application with other concerned nations (Carraz and Harayama, 2018). Today, the topic of ethics remains one of the most controversial topics for the realization of Society 5.0, since different nations and even cultures within a country or area can have different principles and understandings of how to handle and use personal information.

\section{Industry 4.0 V/s Society 5.0}

The idea of Industry 4.0 advocates for an industrial revolution based on manufacturing, as per Deguchi et al. (2020), but says little about how such a revolution will influence the population. By comparison, Society 5.0 reflects extensively on the public effects of technology and on the desire to create a better community, as 
demonstrated by the idea of a people-centric society (Ferreira and Serpa, 2018). Indeed, Germany's Industry 4.0 supports intelligent factories, while Japan's Society 5.0 calls for a super-smart society (Onday, 2019). Furthermore, while all visions support cyber-physical systems deployment, the extent of deployment varies. Despite the aforementioned points, the authors of the study attempt to differentiate the two principles, since Industry 4.0 ultimately calls for a manufacturing-centered technological revolution, but suggests little on how the public may be influenced by such a revolution. On the other hand, Society 5.0 reflects extensively on the public effect of technology and the desire to build a smart sustainable society, as demonstrated by the idea of a people-centric society. In point of fact, Industry 4.0 and Society 5.0 all aim to create a worldwide cyber infrastructure that can serve as a secure space for innovative practises. Society 5.0 is however a more far-reaching term, as it envisages a thorough overhaul in our way of life.

\section{Quadruple Helix Innovation Model (QHIM)}

A society or city is not intelligent if it does not involve all its partners in the processes of decision-making and policy-making or in the processes of creativity (Selada, 2017). The outline of the smart city is focused on the exchange of information and communication at all levels of society. This idea is related conceptually to the outline of the Quadruple Helix Innovation Model (QHIM) (Liljemark, 2004). Here, one new Helix in the process of creativity is added by the QHIM: civil society (Carayannis et al., 2012). In our approach, structures based on the QHIM are spatially contextual and relate to a geographic landscape of innovation in which cities or municipalities play a leading role in collaborating closely with universities, industry and civil society. The outcome is reflected in intelligent policy awareness and creative ideas aimed at addressing social or ecological concerns and adapting answers to the issues facing societies with COVID-19 (Carayannis and Rakhmatullin, 2017; Selada, 2017; Suzic et al., 2020). Any good digital transformation strategy focuses on having a common vision, identifying targets, and setting goals. The problem depends on how states should interfere in relation with other Helixes instead of interceding where they should. At this juncture, the inclusion of foreign networks and collaboration with clusters internationally must also be taken into account when planning the smart governance of a super-smart community.

\section{The human centered efficacy of AI in Society 5.0}

It is no longer in doubt that AI would have a significant effect on the human-focused Society 5.0 outline. The research by Floridi et al. (2018) argues for several big prospects for super-smart societies provided by AI. The basic points in the understanding of human dignity, success, and creativity are addressed: [a] whom we will become (autonomous self-realization); [b] what we can do (human agency); [c] what we can do (individual and social capacities); and [d] how we can connect with each considering global perspective (societal cohesion). Within Society 5.0, AI may encourage self-realization, implying the opportunity for individuals to grow in terms of their traits, desires, future abilities or talents, goals, and life ventures. On the other hand, AI may equate the significance of "Augmented Intelligence" to the effect that engines have had on our lives. The greater the number of individuals who will benefit from the resources and advantages of such a smart organization pool on tap, the healthier our economies will be (Inclezan and Pradanos, 2017). By dramatically changing what people are individually capable of the application of AI technology poses endless opportunities for reinventing communities. Furthermore, with its data-intensive, algorithmic-driven solutions, AI can significantly help to cope with the complexities of teamwork, fostering more cooperation and collaboration in society. Although unintended, AI's predictive capacity and constant nudging should be at the behest of human self-determination and promote social stability, not weakening human integrity or human wellbeing (Chaudhry et al., 2018). Ensuring AI's socially preferable effects is dependent on overcoming the conflict between combining human-centered advantages and minimizing AI's possible damages, in short, simultaneously preventing the misuse and underuse of these technologies (Theodorou and Dignum, 2020). 


\section{Syetemizing AI in Society 5.0 configuration}

Figure-2: Conceptulization of AI triggered Society 5.0 after COVID-19 pandemic

Government

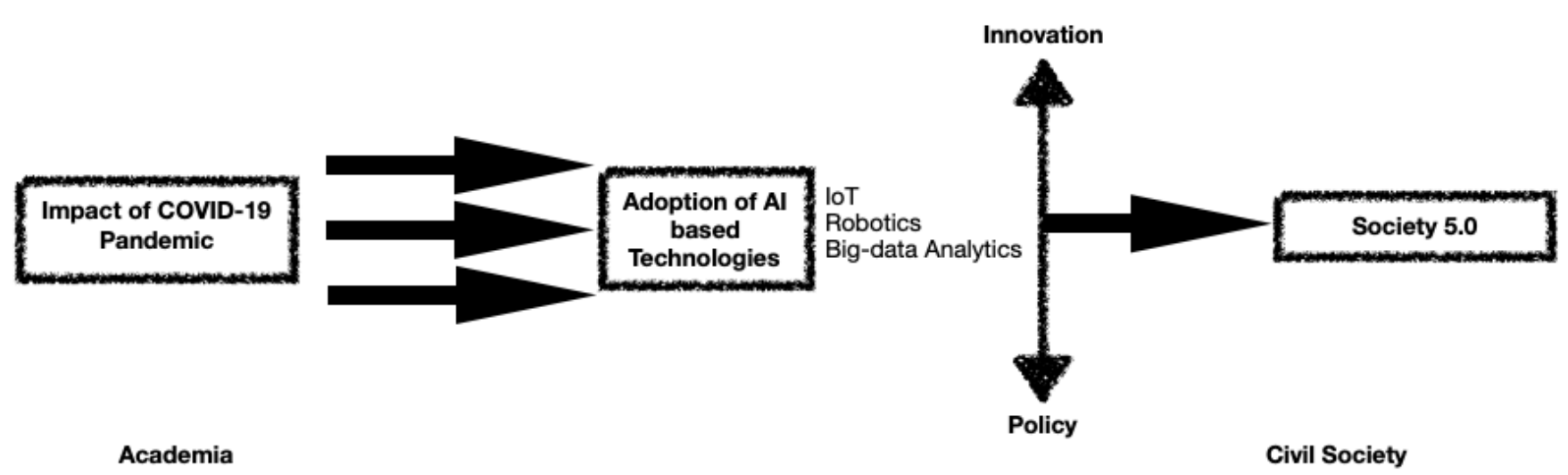

\section{Source: Formulated by authors}

The ability for Artificial Intelligence (AI) is to turn data into value and change all areas of a society (Allam and Dhunny, 2019). In this sense, the future smart world envisaged by the Sustainable Development Goals of the United Nations and Japanese Society 5.0 would entail a digitalization-driven transition of economic and social networks, with artificial intelligence playing a central role. The prospects presented by useful AI technologies for the outline of Society 5.0 are immense (Allam and Jones, 2020). Various types of new services are introduced on the market during this COVID-19 pandemic, and AI implementations have extended to manufacturing, medical, transport, infrastructure, public protection, electricity, education and other fields (Verma and Gustafsson, 2020; Ting et al., 2020). Considering the direction of our analysis, Artificial Intelligence (AI) and other emerging innovations would combine to revolutionise the infrastructure of the Society 5.0 network and create resilient ecosystems (Singh et al., 2020). The AI corresponds to three focus areas of the super-smart society in this configuration, namely wellness, mobility and competitiveness (Shiroishi et al., 2018).

AI carries the power to link to the Internet of Things(IoT) inside Society 5.0 to make smart shops or business setups possible. In robotics, the algorithm behind AI can also be used to extract a number of retail sector technology opportunities (Arsénio et al., 2014). The use of mini robots for grocery shopping, for example, and inventory replenishment in the retail field. Also besides, AI-based warehouse management is also successful. Moreover, via automation, manual functions, including warehouse and product control, machine vision, robots, unmanned vehicles, and AI-supported drones, the retailers of Society 5.0 will eliminate long-term retail back-end costs. More complex logistical functions such as organisational strategy, forecasting and client review can be anticipated by the whole configuration. Moreover, new machine learning and deep learning tools can be provided by AI facet Big-data Analytics (BDA) and delivers the findings directly to decision makers.

From another angle, these innovative developments focused on AI may likely have a subversive influence on the potential growth of the financial sector. Some smart engineering applications will lead the financial sector within the Society 5.0 as a robot adviser, risk management, recognition, smart consumer care, regulatory technology, precision marketing (Mondal and Singh, 2018). Indeed, financial technology's robust growth in recent years is inseparable from the promotion and distribution of BDA and AI. Following the COVID-19 pandemic, the advancement of AI technologies would also facilitate the creative implementation of intelligent healthcare systems within Society 5.0. Since the ageing dynamics in population, the usage of technological products to help healthcare workers in healthcare systems is increasingly concerned. Therefore, many companies affiliated with healthcare are heading towards smart management and operation 
through novel technology (Ahmad et al., 2020). Now-a-days, personal fitness, sports management, diet management, emergency services, tele-consultation, tele-diagnosis, medical care and assistance, remote home care and so on are part of the AI-oriented intelligent healthcare programme spectrum (Hossain et al., 2020; Kaur andMann, 2017). We hope that the AI-based smart health industry will eventually shift towards connected health, customization and family-centered treatment, stressing customer engagement, predictive and environment, taking into account the Society 5.0 outline.

Artificial intelligence development depends on four key elements in the outline of Society 5.0, domain awareness, software design, application development, and hardware manufacture (Lin et al., 2018). Here, rather than an autonomous technology, AI is a catalyst (Mark and Anya, 2019). Companies should also concentrate on how to best use the data to release the benefit of AI with the advent of more and more sophisticated applications. In Society 5.0, in depopulated regions, autonomous vehicles and drones can carry products and services to individuals (Chu, 2020). The successful systemization of AI after the COVID-19 pandemic would make it more interactive and customised for social media and entertainment (Li and Ture, 2020). It will help to establish prevention measures and help law enforcement, such as road tracking, detection of financial crime, unexplained drone tracking, etc. The city can be driven by energy distributed in scalable and autonomous forms to satisfy the individual needs of the inhabitants. The mature production of AI and other emerging technology will in effect, force the original social system to face changes like business and work. As per the above review, we holistically assume that revolutionary AI-based technology has the potential to solve an ageing population, labour scarcity, shortages of energy and services, natural hazards, protection and protection, and all other problems that lead to the creation of an ethical and happy Society 5.0, considering most of the regions of the globe.

\section{Theoretical contributions}

Theoretically, the paper has unpacked AI's core abilities and strengths in the super-smart culture, describing the importance of being intelligent in terms of data, robotics, IoT and technical platforms, and exposing nonbiological intelligence's key societal problems (Stahl and Wright, 2018). The COVID-19 pandemic will in reality, have a short, mid and long-term effects on numerous aspects of society and industry. The brainstorming decision in the philosophical context of the COVID-19 research is an early effort in the field of AI-based super smart society and attainment of SDGs. For experts, the study has established an entirely new model where pandemics will harness sophisticated technology-based configurations for a smart society that supports a human-centered approach (Gardner and Hespanhol, 2018). It also offers more study and negotiations between science and policy on the prospective mechanism between SDGs and Society 5.0.

\section{Practical implications}

The research tracked the configuration of AI in Society 5.0 empirically, highlighting how super-smart social solutions are transforming into autonomous technologies that handle parts of everyday operations without human control (Cugurullo, 2020). The results also serve as a practical blueprint for the introduction of AI technology to Society 5.0 by incorporating open source technologies, constructive legislation and new business models to work together to achieve the SDGs (Islam et al., 2020). This conceptualization will also have a positive influence on the daily lives of people, but the changes it would bring are incremental and not dramatic changes in large part. Furthermore, instead of reducing career opportunities, more jobs and simpler working processes should be created (Rao and Prasad, 2018).

We offer realistic scenarios for policymakers to recognize that the outline of AI-based Society 5.0 has an important role to play in the post-pandemic sustainability process of our environment, our economies and our people. The findings of the study offer decision-makers a range of realistic insights into the problems raised by COVID-19 and the fiscal, social and technical steps and changes that need to be carried out. Through reevaluating laws and regulations and growing their digital presence based on the principle of Society 5.0, the path gained will also assist authority to adapt successfully to resolve future crises. In this sense, the role of digitalization-supporting public policy and private investment would be significant to ensure AI solutions within Society 5.0 leading to SDGs (Fukuyama, 2018; Renda and Laurer, 2020). 


\section{Final thoughts}

Given the probabilities produced from the COVID-19 emergency, our systemic study successfully conceptualised an AI-oriented resilient Society 5.0 configuration. In reality, the central importance of advanced digital technologies aligned with the idea of Society 5.0 contributing to sustainable AI-supported accomplishments is a domain that will generate more interest in the future. Here, the contributions of different stakeholders, especially government, business, university and civil society, on how to design comprehensive socio-political strategies that facilitate the widespread implementation of environmentally and socially preferable AI solutions within Society 5.0 are crucial (Cath et al., 2018). Policies, on the other hand, should ensure that AI is entirely driven towards the advancement of the public good. We need a strong and persuasive understanding of what kind of ethical AI based Society 5.0 we want to build for this reason. Via an unbiased, multinational, multi-stakeholder study mechanism and workshops on AI and Big Data ethics, such awareness will best be accomplished (Fiorini, 2020). Again in Society 5.0 for human prosperity, we agree that advanced emerging technology, practises, sciences, products and services will be tremendously beneficial. In such a larger theme, AI plays a key role. But if we don't pay attention to its growth and controlled use, we are delicate institutions, responsive processes, weak individuals and AI can easily become the elephant in the crystal palace. Governments should take the lead in coordinating this process, because they may have a political mandate to establish AI-based Society 5.0 policy and will be kept responsible for their choices, in a way that is more challenging for the private sector and the science community.

Outbreaks or natural pandemics are a bleak example of how one case can turn a world nightmare into a snowball, but they still have a long history of transforming things for the better. Again as people around the world are grappling with the devastating impact of the COVID-19 emergency on their lives, economies and societies, academics from different perspectives are increasingly determined to make contributions to the war against present and future pandemics. The feeling of belonging to a society that is purely creative, global, supportive, and smartly integrated will help to resolve the challenges of this deep crisis. We expect that this conceptualization provides researchers with guidelines to define super intelligent society configured solutions to address the current global crisis. In reality, the latest pandemic has explicitly released the groundwork for the worldwide implementation of Society 5.0 based on AI-controlled IoT, Big Data, and Robotics that would offer higher economic growth and better quality of life. In this outline, the biggest challenge society faces in the 21 st century can be overcome by mankind and intelligent technology.

Implementing more successful and widespread Society 5.0 programmes will facilitate the retrieval, analysis, storing and distribution of vital info, potentially enhancing the identification and prevention of virus outbreaks while reducing the implementation period when critical steps are taken (Onday, 2019). Furthermore, it accepts the potential to constantly enhance AI-based technology to more reliably and sooner forecast earthquakes, tsunamis and climate change and to respond more quickly when disasters occur. It is clear that with this concept, we have to accept the notion of what Japan is making, or we will literally become a vestige of the technological past. Finally, to ensure that Society 5.0 does not grow into the topdown strategy of a multinational company, it is vital that concrete attempts be taken to involve smaller corporations and groups from diverse backgrounds to really represent the interests of those it claims to concentrate on (Mavrodieva and Shaw, 2020). However, no data is obtained to generalise to other countries or regions since this is a conceptual research paper based on review analysis. On top of that the structure of this study is self-constructed according to prior literature. Generalization is therefore not appropriate for the application. In order to gather more accurate data to prevent bias, prospective experiments need to rely thoroughly on datasets. Also besides, through including adaptive governance or also spiritual/ethical components, the role of COVID-19 for AI-focused Society 5.0 can also be tested logically and empirically by future research expeditions.

\section{References}

[1] Abduljabbar, R., Dia, H., Liyanage, S., \& Bagloee, S. A. (2019). Applications of artificial intelligence in transport: An overview. Sustainability, 11(1), 189. 
[2] Ahmad, M. A., Patel, A., Eckert, C., Kumar, V., \& Teredesai, A. (2020, August). Fairness in Machine Learning for Healthcare. In Proceedings of the 26th ACM SIGKDD International Conference on Knowledge Discovery \& Data Mining (pp. 3529-3530).

[3] Albzeirat, M., Zulkepli, N., \& Qaralleh, H. (2020). A vision to face Covid-19 pandemic and future risks through artificial intelligence. Journal of basic and applied Research in Biomedicine, 6(1), 1520.

[4] Allam, Z., \& Dhunny, Z. A. (2019). On big data, artificial intelligence and smart cities. Cities, 89, 80-91.

[5] Allam, Z., \& Jones, D. S. (2020, March). On the coronavirus (COVID-19) outbreak and the smart city network: universal data sharing standards coupled with artificial intelligence (AI) to benefit urban health monitoring and management. In Healthcare (Vol. 8, No. 1, p. 46). Multidisciplinary Digital Publishing Institute.

[6] Arsénio, A., Serra, H., Francisco, R., Nabais, F., Andrade, J., \& Serrano, E. (2014). Internet of intelligent things: Bringing artificial intelligence into things and communication networks. In Intercooperative collective intelligence: Techniques and applications (pp. 1-37). Springer, Berlin, Heidelberg.

[7] Barnes, S. J. (2020). Information management research and practice in the post-COVID-19 world. International Journal of Information Management, 102175.

[8] Bogler, A., Packman, A., Furman, A., Gross, A., Kushmaro, A., Ronen, A., ... \& Bertuzzo, E. (2020). Rethinking wastewater risks and monitoring in light of the COVID-19 pandemic. Nature Sustainability, 1-10.

[9] Buchanan, B. (2005). A brief history of artificial intelligence. AI Magazine, 26(4), 53-60

[10] Budd, J., Miller, B. S., Manning, E. M., Lampos, V., Zhuang, M., Edelstein, M., ... \& Short, M. J. (2020). Digital technologies in the public-health response to COVID-19. Nature medicine, 1-10.

[11] Budd, J., Miller, B. S., Manning, E. M., Lampos, V., Zhuang, M., Edelstein, M., ... \& Short, M. J. (2020). Digital technologies in the public-health response to COVID-19. Nature medicine, 1-10.

[12] Carayannis, E. G., \& Rakhmatullin, R. (2014). The quadruple/quintuple innovation helixes and smart specialisation strategies for sustainable and inclusive growth in Europe and beyond. Journal of the Knowledge Economy, 5(2), 212-239.

[13] Carayannis, E. G., Barth, T. D., \& Campbell, D. F. (2012). The Quintuple Helix innovation model: global warming as a challenge and driver for innovation. Journal of innovation and entrepreneurship, 1(1), 1-12.

[14] Carraz, R., \& Harayama, Y. (2018). Japan's Innovation Systems at the Crossroads: Society 5.0. Digital Asia, 33-45.

[15] Cath, C., Wachter, S., Mittelstadt, B., Taddeo, M., \& Floridi, L. (2018). Artificial intelligence and the 'good society': the US, EU, and UK approach. Science and engineering ethics, 24(2), 505-528.

[16] Chaudhry, J., Pathan, A. S. K., Rehmani, M. H., \& Bashir, A. K. (2018). Threats to critical infrastructure from AI and human intelligence. The Journal of Supercomputing, 74(10), 4865-4866.

[17] Chu, K. F. (2020). Artificial intelligence empowered autonomous vehicles on-demand system for smart cities. HKU Theses Online (HKUTO).

[18] Collin, S. O., Johansson, U., Svensson, K., \& Ulvenblad, P. O. (1996). Market Segmentation in Scientific Publications: Research Patterns in American vs European Management Journals1. British Journal of Management, 7(2), 141-154. 
[19] Cugurullo, F. (2020). Urban artificial intelligence: From automation to autonomy in the smart city. Front. Sustain. Cities, 2, 38 (1-14).

[20] Deguchi, A., Hirai, C., Matsuoka, H., Nakano, T., Oshima, K., Tai, M., \& Tani, S. (2020). What Is Society 5.0?. In Society 5.0 (pp. 1-23). Springer, Singapore.

[21] DeWit, A., Shaw, R., \& Djalante, R. (2020). An integrated approach to sustainable development, National Resilience, and COVID-19 responses: The case of Japan. International Journal of Disaster Risk Reduction, 101808.

[22] Di Vaio, A., Palladino, R., Hassan, R., \& Escobar, O. (2020). Artificial intelligence and business models in the sustainable development goals perspective: A systematic literature review. Journal of Business Research, 121, 283-314.

[23] Dobrev, D. (2012). A definition of artificial intelligence. arXiv preprint arXiv:1210.1568.

[24] Donthu, N., \& Gustafsson, A. (2020). Effects of COVID-19 on business and research. Journal of business research, 117, 284-289.

[25] Fagerberg, J., Fosaas, M., \& Sapprasert, K. (2012). Innovation: Exploring the knowledge base. Research policy, 41(7), 1132-1153.

[26] Ferreira, C. M., \& Serpa, S. (2018). Society 5.0 and Social Development. Management and Organizational Studies 1(5), 26-31.

[27] Floridi, L., Cowls, J., Beltrametti, M., Chatila, R., Chazerand, P., Dignum, V., ... \& Schafer, B. (2018). AI4People - an ethical framework for a good AI society: opportunities, risks, principles, and recommendations. Minds and Machines, 28(4), 689-707.

[28] Fiorini, R. A. (2020). Computational Intelligence From Autonomous System to Super-Smart Society and Beyond. International Journal of Software Science and Computational Intelligence (IJSSCI), 12(3), 1-13.

[29] Fukuda, K. (2020). Science, technology and innovation ecosystem transformation toward society 5.0. International Journal of Production Economics, 220, 107460.

[30] Fukuyama, M. (2018). Society 5.0: Aiming for a New Human-centered Society. Japan SPOTLIGHT, 27 (Society 5.0), 47-50.

[31] Gardner, N., \& Hespanhol, L. (2018). SMLXL: Scaling the smart city, from metropolis to individual. City, Culture and Society, 12, 54-61.

[32] Gartner Inc. (2020). Reset Your Business Strategy Amid COVID-19. NY. Retrieved from https://www.gartner.com/en/newsroom/press-releases/2020-10-01-gartner-survey-revels-66-percentof-orgnizations-increased-or-did-not-change-ai-investments-since-the-onset-of-covid19?fbclid=IwAR1HwKyddHLsmsLr0iE895_3ktE6n2T2ynS5_VhYhkAEyxU7CDRBx1T7PAQ

[33] Goralski, M. A., \& Tan, T. K. (2020). Artificial intelligence and sustainable development. The International Journal of Management Education, 18(1), 100330.

[34] Greenspan, H., Estépar, R. S. J., Niessen, W. J., Siegel, E., \& Nielsen, M. (2020). Position paper on COVID-19 imaging and AI: From the clinical needs and technological challenges to initial AI solutions at the lab and national level towards a new era for AI in healthcare. Medical image analysis, 66, 101800 .

[35] Gupta, M., Abdelsalam, M., \& Mittal, S. (2020). Enabling and enforcing social distancing measures using smart city and its infrastructures: a COVID-19 Use case. arXiv preprint arXiv:2004.09246.

[36] Hanif, M. I., \& Iftikhar, L. (2020). Post COVID-19 Industrial Revolution 5.0. The dawn of Cobot, Chipbot and Curbot. Pakistan Journal of Surgery and Medicine, 1(2), 122-126. 
[37] Hart, C. (2001). Doing a literature search: a comprehensive guide for the social sciences. Sage.

[38] Herath, T., \& Herath, H. S. (2020). Coping with the New Normal Imposed by the COVID-19 Pandemic: Lessons for Technology Management and Governance. Information Systems Management, 1-7.

[39] Holroyd, C. (2020). Technological innovation and building a 'super smart'society: Japan's vision of society 5.0. Journal of Asian Public Policy, 1-14.

[40] Hossain, M. S., Muhammad, G., \& Guizani, N. (2020). Explainable AI and mass surveillance system-based healthcare framework to combat COVID-I9 like pandemics. IEEE Network, 34(4), 126-132.

[41] Hu, R. (2020). Reinventing community in COVID-19: a case in Canberra, Australia. SocioEcological Practice Research, 2(3), 237-241.

[42] Huynh, E., Hosny, A., Guthier, C., Bitterman, D. S., Petit, S. F., Haas-Kogan, D. A., ... \& Mak, R. H. (2020). Artificial intelligence in radiation oncology. Nature Reviews Clinical Oncology, 1-11.

[43] IDC. (2020). Worldwide Artificial Intelligence Spending Guide. Framingham. Retrieved from https://www.idc.com/getdoc.jsp?containerId=prUS46794720.

[44] Inclezan, D., \& Pradanos, L. I. (2017). A critical view on smart cities and AI. Journal of Artificial Intelligence Research, 60, 681-686.

[45] Islam, A., Anum, K., Dwidienawati, D., Wahab, S., \& Abdul Latiff, A. (2020). Building a post COVID-19 Configuration between Internet of Things (IoT) and Sustainable Development Goals (SDGs) for Developing Countries. Journal Of Arts \& Social Sciences, 4(1), 45-58.

[46] Islam,A., \& Wahab, S.(2020).Does Covid-19 help Sustainable Business Configuration through BigData Analytics (BDA)?.Journal Of Arts \& Social Sciences, 4(1), 18-30.

[47] Jahn, T., Bergmann, M., \& Keil, F. (2012). Transdisciplinarity: Between mainstreaming and marginalization. Ecological Economics, 79, 1-10.

[48] Jaiswal, R., Agarwal, A., \& Negi, R. (2020). Smart solution for reducing the COVID-19 risk using smart city technology. IET Smart Cities, 2(2), 82-88.

[49] Jetten, J. (2020). Together apart: The psychology of Covid-19. Sage.

[50] Kaur, J., \& Mann, K. S. (2017, October). AI Based HealthCare Platform for Real Time, Predictive and Prescriptive Analytics. In International Conference on Computing, Analytics and Networks (pp. 138-149). Springer, Singapore.

[51] Ku, C.-H., \& Leroy, G. (2014). A decision support system: Automated crime report analysis and classification for e-government. Government Information Quarterly, 31(4), 534-544.

[52] Lachman, R., \& Joffe, M. (2020). Applications of Artificial Intelligence in Media and Entertainment. In Analyzing Future Applications of AI, Sensors, and Robotics in Society (pp. 201-220). IGI Global..

[53] Letaief, K. B., Chen, W., Shi, Y., Zhang, J., \& Zhang, Y. J. A. (2019). The roadmap to 6G: AI empowered wireless networks. IEEE Communications Magazine, 57(8), 84-90.

[54] Li, W., \& Ture, F. (2020, July). Auto-annotation for Voice-enabled Entertainment Systems. In Proceedings of the 43rd International ACM SIGIR Conference on Research and Development in Information Retrieval (pp. 1557-1560). IEEE.

[55] Liljemark, T. (2004). Innovation policy in Canada. Strategy and realities. Swedish Institute for Growth Policy Studies. 
[56] Lin, Y. C., Lin, Y. T., \& Huang, P. S. (2018, October). Future prospects of artificial intelligence and how it drive to smart society. In 2018 International Conference on Orange Technologies (ICOT) (pp. 1-3). IEEE.

[57] Mark, R., \& Anya, G. (2019). Ethics of using smart city AI and Big Data: The case of four large European cities. The ORBIT Journal, 2(2), 1-36.

[58] Masuhr, N. (2019). AI in Military Enabling Applications. CSS Analyses in Security Policy, 251.

[59] Mavrodieva, A. V., \& Shaw, R. (2020). Disaster and Climate Change Issues in Japan's Society 5.0A Discussion. Sustainability, 12(5), 1893.

[60] Mavrodieva, A. V., \& Shaw, R. (2020). Disaster and Climate Change Issues in Japan's Society 5.0A Discussion. Sustainability, 12(5), 1893.

[61] Max-Neef, M. A. (2005). Foundations of transdisciplinarity. Ecological economics, 53(1), 5-16.

[62] McArthur, D., Lewis, M., \& Bishary, M. (2005). The roles of artificial intelligence in education: current progress and future prospects. Journal of Educational Technology, 1(4), 42-80.

[63] Miner, A. S., Laranjo, L., \& Kocaballi, A. B. (2020). Chatbots in the fight against the COVID-19 pandemic. npj Digital Medicine, 3(1), 1-4.

[64] Mondal, A., \& Singh, A. (2018, December). Emerging Technologies and Opportunities for Innovation in Financial Data Analytics: A Perspective. In International Conference on Big Data Analytics (pp. 126-136). Springer, Cham.

[65] Muhthar, A., Ahmad, A. R., \& Awang, M. M. (2020). Reinforcement Historical Thinking in Society 5.0 During Covid-19 Outbreak. Journal of Talent Development and Excellence, 12(1), 5843-5851.

[66] Muhthar, A., Ahmad, A. R., \& Awang, M. M. (2020). Reinforcement Historical Thinking in Society 5.0 During Covid-19 Outbreak. Journal of Talent Development and Excellence, 12(1), 5843-5851.

[67] Murad, K. (2014). Key to al-Baqarah: The Longest Surah of the Qur'an (Vol. 1). Kube Publishing Ltd.

[68] Naudé, W. (2020). Artificial intelligence vs COVID-19: limitations, constraints and pitfalls. Ai \& Society, 1.

[69] Nazir, A., Azhar, A., Nazir, U., Liu, Y. F., Qureshi, W. S., Chen, J. E., \& Alanazi, E. (2020). The rise of 3D Printing entangled with smart computer aided design during COVID-19 era. Journal of Manufacturing Systems.

[70] Onday, O. (2019). Japan's society 5.0: Going beyond Industry 4.0. Business and Economics Journal, 10(2), 1-6.

[71] Panigutti, C., Perotti, A., \& Pedreschi, D. (2020, January). Doctor XAI: an ontology-based approach to black-box sequential data classification explanations. In Proceedings of the 2020 Conference on Fairness, Accountability, and Transparency (pp. 629-639).

[72] Polat, L., \& Erkollar, A. (2020, September). Industry 4.0 vs. Society 5.0. In The International Symposium for Production Research (pp. 333-345). Springer, Cham.

[73] Rao, S. K., \& Prasad, R. (2018). Impact of 5G technologies on smart city implementation. Wireless Personal Communications, 100(1), 161-176.

[74] Rochman, G. P., Chofyan, I., \& Sakti, F. (2020). Understanding the smart society in rural development. E\&ES, 447(1), 012016. 
[75] Selada, C. (2017). Smart cities and the quadruple helix innovation systems conceptual framework: The case of Portugal. In The Quadruple Innovation Helix Nexus (pp. 211-244). Palgrave Macmillan, New York.Systemizing AI in Society 5.0 outline

[76] Seuring, S., \& Müller, M. (2008). From a literature review to a conceptual framework for sustainable supply chain management. Journal of cleaner production, 16(15), 1699-1710.

[77] Sharp, L. (2020). Society 5.0: A brave new world. Impact, 2020(2), 2-3.

[78] Shiroishi, Y., Uchiyama, K., \& Suzuki, N. (2018). Society 5.0: For human security and wellbeing. Computer, 51(7), 91-95.

[79] Simmons, A. B., \& Chappell, S. G. (1988). Artificial intelligence-definition and practice. IEEE Journal of Oceanic Engineering, 13(2), 14-42.

[80] Singh, S., Sharma, P. K., Yoon, B., Shojafar, M., Cho, G. H., \& Ra, I. H. (2020). Convergence of blockchain and artificial intelligence in IoT network for the sustainable smart city. Sustainable Cities and Society, 63, 102364.

[81] Sokolov, M. (2020). Decision Making and Risk Management in Biopharmaceutical EngineeringOpportunities in the Age of Covid-19 and Digitalization. Industrial \& Engineering Chemistry Research, 59(40), 17587-17592.

[82] Stahl, B. C., \& Wright, D. (2018). Ethics and privacy in AI and big data: Implementing responsible research and innovation. IEEE Security \& Privacy, 16(3), 26-33.

[83] Sun, T. Q., \& Medaglia, R. (2019). Mapping the challenges of Artificial Intelligence in the public sector: Evidence from public healthcare. Government Information Quarterly, 36(2), 368-383.

[84] Suzic, B., Ulmer, A., \& Schumacher, J. (2020, June). Complementarities and Synergies of Quadruple Helix Innovation Design in Smart City Development. In 2020 Smart City Symposium Prague (SCSP) (pp. 1-7). IEEE.

[85] Tashiro, A., \& Shaw, R. (2020). COVID-19 pandemic response in Japan: what is behind the initial flattening of the curve?. Sustainability, 12(13), 5250.

[86] The Business Research Company. (2020). Artificial Intelligence Global Market Report 2020-30: COVID-19 Growth and Change. Global. Retrieved from https://www.researchandmarkets.com/reports/5050683/artificial-intelligence-global-market-report2020?utm source=dynamic\&utm medium=GNOM\&utm code=f2kzld\&utm campaign=1397617++Global+Artificial+Intelligence+Market+Report+(2020+to+2030)+-+COVID$19+$ Growth+and+Change\&utm_exec=jamu273gnomd

[87] Theodorou, A., \& Dignum, V. (2020). Towards ethical and socio-legal governance in AI. Nature Machine Intelligence, 2(1), 10-12.

[88] Ting, D. S. W., Carin, L., Dzau, V., \& Wong, T. Y. (2020). Digital technology and COVID19. Nature medicine, 26(4), 459-461.

[89] Ting, D. S. W., Carin, L., Dzau, V., \& Wong, T. Y. (2020). Digital technology and COVID19. Nature medicine, 26(4), 459-461.

[90] Vaishya, R., Javaid, M., Khan, I. H., \& Haleem, A. (2020). Artificial Intelligence (AI) applications for COVID-19 pandemic. Diabetes \& Metabolic Syndrome: Clinical Research \& Reviews. 
[91] Verma, S., \& Gustafsson, A. (2020). Investigating the emerging COVID-19 research trends in the field of business and management: A bibliometric analysis approach. Journal of Business Research, 118, 253-261.

[92] Whitelaw, S., Mamas, M. A., Topol, E., \& Van Spall, H. G. (2020). Applications of digital technology in COVID-19 pandemic planning and response. The Lancet Digital Health.

[93] Wirtz, B. W., Weyerer, J. C., \& Geyer, C. (2019). Artificial intelligence and the public sectorapplications and challenges. International Journal of Public Administration, 42(7), 596-615.

[94] Woo, J. J. (2020). Policy capacity and Singapore's response to the COVID-19 pandemic. Policy and Society, 39(3), 345-362.

[95] Ye, J. (2020). The role of health technology and informatics in a global public health emergency: practices and implications from the COVID-19 pandemic. JMIR Medical Informatics, 8(7), e19866. 